\title{
Évaluation hémodynamique précoce par échocardiographie ciblée des patients en sepsis ou choc septique (Sepsis-3) au service d'urgence
}

\author{
Description of the Early Hemodynamic Profile of Septic Patients Assessed by Focused \\ Echocardiography in the Emergency Department
}

\author{
T. Lafon $\cdot$ P. Feydeau $\cdot$ A. Baisse $\cdot$ V. Bigrat $\cdot$ C. Raynaud $\cdot$ M. Pihan $\cdot$ M. Goudelin $\cdot$ B. Evrard $\cdot$ C. Vallejo $\cdot$ \\ H. Hani Karam · P. Vignon \\ Reçu le 9 avril 2020 ; accepté le 11 août 2020 \\ (C) SFMU et Lavoisier SAS 2020
}

\begin{abstract}
Résumé Introduction : L'identification du profil hémodynamique des patients septiques au service d'urgence (SU) pourrait permettre d'adapter individuellement les recommandations de la Surviving Sepsis Campaign (SSC). L'objectif de cette étude était de décrire le profil hémodynamique précoce par échocardiographie ciblée chez les patients en sepsis et leur impact thérapeutique.

Méthode : Étude prospective monocentrique incluant les patients septiques (score qSOFA [quick Sepsis-related Organ Failure Assessment] $\geq 2$ ) sur une période de 30 mois. L'échocardiographie était réalisée par des médecins urgentistes de niveau échographie clinique en médecine d'urgence 1 afin de déterminer le profil hémodynamique (hypovolémie, vasoplégie avec hyperkinésie, insuffisance ventriculaire ou profil « normal ») dès le sepsis identifié au SU. L'impact thérapeutique était défini par le nombre de patients pour lesquels l'échocardiographie modifiait le traitement de la SSC.
\end{abstract}

T. Lafon $(\bowtie) \cdot$ C. Vallejo $\cdot$ P. Vignon

Inserm CIC 1435, centre hospitalier universitaire Dupuytren,

2, avenue Martin-Luther-King, F-87042 Limoges, France

e-mail : Thomas.LAFON@chu-limoges.fr

T. Lafon $\cdot$ P. Feydeau $\cdot$ A. Baisse $\cdot$ V. Bigrat $\cdot$ C. Raynaud

M. Pihan · C. Vallejo · H. Hani Karam

Service d'urgences, CHU Dupuytren,

2, avenue Martin-Luther-King, F-87042 Limoges, France

M. Goudelin · B. Evrard · P. Vignon

Réanimation polyvalente, CHU Dupuytren,

2, avenue Martin-Luther-King, F-87042 Limoges, France

P. Vignon

Inserm UMR 1092, CHU Dupuytren,

2, avenue Martin-Luther-King, F-87042 Limoges, France

Faculté de médecine, université de Limoges,

2, rue du Docteur-Marcland, F-87025 Limoges cedex, France
Résultats : Quatre-vingt-un patients (44 sepsis, 37 chocs septiques) ont été étudiés (âge moyen : $70 \pm 15$ ans ; hommes $[n=47,58 \%] ;$ SOFA $=5,3 \pm 2,9$; lactates $=4,6 \pm$ $4,2 \mathrm{mmol} / 1$; admission en réanimation $[n=30,37 \%]$; mortalité j28 $[n=28,35 \%])$ après un remplissage vasculaire médian de $500 \mathrm{ml}$ (écart interquartile : 250-1 $500 \mathrm{ml}$ ). Les profils hémodynamiques dominants étaient l'hypovolémie $(n=54,66 \%)$, parfois associée à une vasoplégie $(n=26,32 \%)$ et l'insuffisance ventriculaire $(n=21,26 \%)$. L'impact thérapeutique concernait 21 patients ( $26 \%$ ), dont un arrêt précoce du remplissage vasculaire après un volume de $850 \pm 740 \mathrm{ml}$ chez huit patients.

Conclusion : La dysfonction ventriculaire précoce est présente chez plus de $20 \%$ des patients septiques. L'impact pronostique d'une évaluation échocardiographique dès l'identification du sepsis au SU reste à déterminer.

Mots clés Sepsis · Choc septique · Échocardiographie Défaillance hémodynamique $\cdot$ Service d'urgence

Abstract Introduction: Identification of hemodynamic profile of septic patients in the Emergency Department (ED) could allow early tailoring recommendations of the Surviving Sepsis Campaign (SSC). The study aims at describing early hemodynamic profiles encountered in septic patients using focused echocardiography and their therapeutic impact.

Methods: We prospectively enrolled adult patients admitted to the ED with sepsis (qSOFA [quick Sepsis-related Organ Failure Assessment] $\geq 2$ ) during a 30-month period. Echocardiography was performed by emergency physicians trained to Critical Care Ultrasound level 1. Investigators determined the hemodynamic profile based on simple yet robust criteria (hypovolemia, vasoplegia with hyperdynamic state, ventricular failure, or apparently normal profile). Therapeutic impact was defined by the number of patients for whom 
early echocardiography modified SSC-based therapy.

Results: Echocardiography was performed in 81 patients (mean age: $70 \pm 15$ years; men $[N=47,58 \%]$; SOFA $=$ $5.3 \pm 2.9$; lactates $=4.6 \pm 4.2 \mathrm{mmol} / \mathrm{l}$; Intensive Care Unit admission $(N=30,38 \%$ ]; Day-28 mortality $[N=28,35 \%])$ after a median fluid loading of $500 \mathrm{ml}$ (interquartile range: $500-1500 \mathrm{ml}$ ). Forty four patients had sepsis and 37 sustained septic shock. Hemodynamic profiles were hypovolemia in 54 patients $(67 \%)$, potentially associated with vasoplegia in 26 patients $(32 \%)$, and ventricular failure in 21 patients $(26 \%)$. Ongoing therapy was altered based on early echocardiographic assessment in $26 \%$ of cases $(N=21)$, including discontinuation of fluid resuscitation after a median volume of $850 \pm 740 \mathrm{ml}$ in 8 patients.

Conclusion: Early ventricular dysfunction involved more than $20 \%$ of septic patients in the ED. Whether tailored therapeutic management guided by focused echocardiographic assessment impacts patient-centered outcome remains to be determined.

Keywords Sepsis - Septic shock - Echocardiography · Hemodynamic failure $\cdot$ Emergency medicine

\section{Introduction}

L'Organisation mondiale de la santé a fait du sepsis une priorité mondiale en termes de santé publique et a adopté une stratégie visant à améliorer son diagnostic et sa prise en charge thérapeutique dès le service d'urgence (SU) [1]. Les dernières recommandations de la Surviving Sepsis Campaign (SSC) proposent de réaliser le prélèvement d'hémocultures, la mesure de la lactatémie, l'administration d'antibiotiques et un remplissage vasculaire de $30 \mathrm{ml} / \mathrm{kg}$ de cristalloïdes en cas d'hypotension artérielle dans l'heure qui suit l'identification du sepsis [2]. Cependant, le remplissage vasculaire précoce n'est pas systématiquement associé à une amélioration du pronostic du sepsis contrairement à la précocité du traitement antibiotique et peut même s'avérer délétère en entraînant une surcharge hydrosodée tissulaire $[3,4]$. Ainsi, la SSC préconise d'investiguer une approche personnalisée du remplissage vasculaire afin de définir pour chaque patient le volume adéquat à administrer selon le mécanisme de défaillance hémodynamique initial [5].

La défaillance circulatoire du sepsis combine de manière variable et évolutive : hypovolémie, vasoplégie et dysfonction cardiaque [6]. L'échocardiographie est actuellement conseillée en première intention pour identifier l'origine d'une défaillance circulatoire, dont le choc septique reste la première cause [7]. Lorsqu'elle est réalisée en réanimation, elle modifie le traitement guidé par les recommandations de la SSC dans environ $50 \%$ des cas [8]. Néanmoins, le traite- ment de la défaillance circulatoire débute bien en amont, dès l'admission du patient au SU, et l'apport de l'évaluation hémodynamique par échocardiographie ciblée à ce stade précoce est inconnu.

L'objectif de notre étude est d'évaluer le profil hémodynamique des patients en sepsis ou en choc septique au SU par échocardiographie ciblée précoce et de mesurer son impact potentiel sur le traitement initial recommandé par la SSC.

\section{Méthodes}

Nous avons colligé prospectivement une série de cas au CHU de Limoges de janvier 2017 à juillet 2019 concernant des patients pour lesquels un sepsis était identifié au cours de leur passage au SU. Les critères d'éligibilité comprenaient : une infection suspectée ou confirmée associée à un score qSOFA (quick Sepsis-related Organ Failure Assessment) supérieur ou égal à 2 points (deux parmi les trois critères suivants : fréquence respiratoire supérieure ou égale à $22 / \mathrm{min}$, pression artérielle systolique inférieure ou égale à $100 \mathrm{mmHg}$ et encéphalopathie reflétée par un score de Glasgow < 15) [9]. Le protocole a été approuvé par le comité d'éthique local du CHU de Limoges (numéro : 210-2016-24).

L'évaluation échocardiographique était réalisée au plus tôt suivant l'identification du sepsis (quel que soit le traitement déjà administré), en sus de la prise en charge habituelle du patient septique fondée sur les recommandations de la SSC, par un médecin urgentiste disponible formé au niveau échographie clinique en médecine d'urgence 1 (ECMU 1), selon un plan d'évaluation hémodynamique prédéfini. L'examen comprenait systématiquement une coupe parasternale grand et petit axes, une coupe apicale quatre cavités et une coupe sous-costale incluant l'exploration de la veine cave inférieure (VCI) [10]. L'investigateur répondait à une liste limitée de questions cliniques standardisées binaires concernant [11] : la taille (mesure du diamètre télédiastolique) et l'évaluation visuelle de la fonction systolique (fraction d'éjection du ventricule gauche [FEVG]), le caractère homogène ou non de l'épaississement myocardique du VG, la taille (mesure du rapport des diamètres télédiastoliques $\mathrm{VD} / \mathrm{VG}$ ) et la fonction systolique globale du ventricule droit (VD), la présence ou non d'un épanchement péricardique ou d'une tamponnade, la taille (mesure du diamètre téléexpiratoire entre l'abouchement du vaisseau dans l'oreillette droite et de la veine hépatique supérieure) et les variations inspiratoires de la VCI chez les patients en ventilation spontanée (une réduction du diamètre de plus de $50 \%$ en inspiration définissant le collapsus inspiratoire) [12] et la présence d'une fuite valvulaire massive aiguë en Doppler couleur. 
Les profils hémodynamiques étaient identifiés selon les critères récemment décrits chez les patients en choc septique, à savoir [13] : hypovolémie, insuffisance VG, insuffisance VD, vasoplégie avec VG hyperkinétique, tamponnade et insuffisance valvulaire mitrale ou aortique massive (Tableau 1). Le cœur pulmonaire aigu était défini par l'association d'une dilatation du VD en grand axe (rapport des diamètres télédiastoliques $\mathrm{VD} / \mathrm{VG}>0,8$ ) [13] et d'une cinétique paradoxale du septum interventriculaire en petit axe. Ces profils étaient établis à la fois sur des données cliniques et échocardiographiques.

L'impact thérapeutique potentiel était défini par le nombre de patients pour lesquels l'échocardiographie modifiait le traitement fondé sur les recommandations de la SSC au moment de l'évaluation hémodynamique : poursuite du remplissage vasculaire au-delà de $30 \mathrm{ml} / \mathrm{kg}$ (profil hypovolémique), instauration précoce d'un traitement vasopresseur (profil vasoplégique) ou, à l'inverse, arrêt prématuré du remplissage vasculaire (avant $30 \mathrm{ml} / \mathrm{kg}$ ), voire introduction d'un traitement inotrope positif (profil de dysfonction ventriculaire responsable de signes d'hypoperfusion tissulaire).

\section{Méthode statistique}

Les résultats sont présentés sous forme d'effectifs, de proportions, de moyennes avec écart-type ou de médianes avec $25^{\mathrm{e}}$ et $75^{\mathrm{e}}$ percentiles. Afin d'avoir une cohorte représentative de la population étudiée et d'obtenir une précision de $10 \%$, on a estimé que la prévalence de l'insuffisance VG au SU serait de l'ordre de $20 \%$, proportion inférieure à celle rapportée habituellement dans les cohortes de patients en choc septique en réanimation $[14,15]$. Pour garantir un risque alpha de $5 \%$, le nombre minimum de patients à évaluer était de 61. Les tests statistiques ont été appliqués en formulation bilatérale. La vérification des normalités des distributions des variables quantitatives a été réalisée par la méthode de Shapiro-Wilk. Les variables catégorielles étaient comparées par le test $\mathrm{du}_{\mathrm{Chi}}{ }^{2}$ ou le test exact de Fischer selon la taille de l'effectif. Les variables quantitatives continues de distribution normale étaient comparées par test $t$ de Student ou avec le test non paramétrique $U$ de MannWhitney pour les variables ne suivant pas une distribution normale, et par analyse de variance avec statistique de Fisher

Tableau 1 Critères échocardiographiques des différents profils hémodynamiques [13]

\begin{tabular}{|c|c|}
\hline Profils hémodynamiques & Critères échocardiographiques ${ }^{a}$ \\
\hline Hypovolémie sévère & $\begin{array}{l}\text { - Ventricules de petite taille } \\
\text { - Diamètre } \mathrm{VCI}<15 \mathrm{~mm} \text { avec variation respiratoire marquée (réduction de la taille }>50 \% \\
\text { en ventilation spontanée) } \\
\text { - FEVG normale ou augmentée (si association avec une vasoplégie) }\end{array}$ \\
\hline Vasoplégie (profil hyperkinétique) & $\begin{array}{l}\text { - VG hyperkinétique } \\
\text { - } \pm \text { Disparition quasi complète de la cavité VG en fin de systole } \\
\text { - } \pm \text { Signes associés d'hypovolémie sévère }\end{array}$ \\
\hline Insuffisance ventriculaire gauche & $\begin{array}{l}\text { - Dysfonction systolique globale du VG } \\
\text { - } \pm \text { Épaississement myocardique hétérogène (anomalie de contraction segmentaire) } \\
\text { - } \pm \text { Dilatation de la cavité VG (insuffisance cardiaque chronique) } \\
\text { - } \pm \text { Dilatation de la VCI ( }>25 \mathrm{~mm} \text { ) avec diminution ou disparition du collapsus inspiratoire } \\
\text { (ventilation spontanée) }\end{array}$ \\
\hline Insuffisance ventriculaire droite & $\begin{array}{l}\text { - Dilatation du VD avec un rapport VD/VG télédiastolique }>0,8 \\
\text { - } \pm \text { Cœur pulmonaire aigu (association d'une dilatation VD en grand axe et d'un septum } \\
\text { paradoxal en petit axe) } \\
\text { - } \pm \text { Dilatation de la VCI ( }>25 \mathrm{~mm} \text { ) avec diminution ou disparition du collapsus inspiratoire } \\
\text { (ventilation spontanée) }\end{array}$ \\
\hline Insuffisance valvulaire massive & $\begin{array}{l}\text { - Jet régurgitant (mitral ou aortique) large en cartographie Doppler couleur (à l'origine, } \\
\text { en surface) } \\
\text { - FEVG normale ou augmentée (surcharge de volume aiguë) } \\
\text { - Taille de la cavité VG normale (pas de dilatation en cas de régurgitation aiguë) }\end{array}$ \\
\hline Tamponnade & $\begin{array}{l}\text { - Épanchement péricardique liquidien compressif sur les cavités cardiaques droites } \\
\text { - Dilatation VCI (>25 mm) avec diminution ou disparition du collapsus inspiratoire } \\
\text { (ventilation spontanée) }\end{array}$ \\
\hline
\end{tabular}


pour comparer les moyennes de plusieurs groupes (rapport des variances inter- et intraclasses).

\section{Résultats}

Pendant la période d'étude, 285 patients septiques avec un score qSOFA supérieur ou égal à 2 ont été admis au SU. Parmi eux, 81 patients (hommes : $n=47$; âge moyen : 70 \pm 15 ans ; SOFA moyen : 5,3 $\pm 2,9$; lactatémie moyenne : $4,7 \pm 4,3 \mathrm{mmol} / \mathrm{l}, \mathrm{FEVG}<50 \% n=5$ ) ont été évalués précocement par échocardiographie ciblée pour sepsis $(n=44)$ ou pour choc septique $(n=37)$. La prévalence de chaque critère du score qSOFA était de $89 \%$ pour la polypnée $(n=72), 62 \%$ pour la confusion $(n=50)$ et $84 \%$ pour l'hypotension artérielle $(n=68)$. L'origine de l'infection était principalement pulmonaire $(n=30,37 \%)$, urinaire ( $n=20,25 \%)$ et digestive $(n=20,25 \%)$. Parmi les dysfonctions d'organes, la défaillance hémodynamique était la plus fréquente $(n=68,84 \%)$, suivie de la défaillance respiratoire $(n=54,67 \%)$, rénale $(n=47,58 \%)$, neurologique ( $n=47,58 \%$ ), hématologique $(n=29,36 \%)$ et hépatique $(n=26,32 \%)$. Trente et un patients $(38 \%)$ ont été hospitalisés en réanimation et la mortalité à 28 jours était de $35 \%$ $(n=28)$ (Tableau 2).

L'échocardiographie a été réalisée après un volume médian de remplissage vasculaire de $500 \mathrm{ml}$ : [250-1500], et toujours avant d'avoir administré au patient la totalité des $30 \mathrm{ml} / \mathrm{kg}$. Le principal mécanisme de défaillance hémodynamique était l'hypovolémie chez 54 patients (66\%), la

Tableau 2 Caractéristiques générales de la population d'étude et description des profils hémodynamiques identifiés par échocardiographie

Population d'étude $(n=81)$

\begin{tabular}{|c|c|}
\hline \multicolumn{2}{|l|}{ Caractéristiques cliniques et biologiques } \\
\hline Âge (années) & $70 \pm 15$ \\
\hline Homme & $47(58)$ \\
\hline \multicolumn{2}{|l|}{ Sévérité } \\
\hline Sepsis & $44(54)$ \\
\hline Choc septique & $37(46)$ \\
\hline \multicolumn{2}{|l|}{ Paramètres hémodynamiques } \\
\hline Fréquence cardiaque (/min) & $107 \pm 25$ \\
\hline Pression artérielle moyenne $(\mathrm{mmHg})$ & $67 \pm 19$ \\
\hline Saturation $\mathrm{O}_{2}$ & $94 \pm 5$ \\
\hline Remplissage vasculaire $^{\mathrm{a}}(\mathrm{ml})$ & $500[250-1500$ \\
\hline \multicolumn{2}{|l|}{ Biologie } \\
\hline $\mathrm{pH}$ & $7,34 \pm 0,13$ \\
\hline Lactate $(\mathrm{mmol} / \mathrm{l})$ & $4,6 \pm 4,2$ \\
\hline Créatinine $(\mu \mathrm{mol} / \mathrm{l})$ & $148 \pm 88$ \\
\hline Plaquettes $\left(10^{9} \mathrm{G} / \mathrm{l}\right)$ & $205 \pm 120$ \\
\hline Hémoglobine (g/dl) & $12,7 \pm 2,3$ \\
\hline Score SOFA moyen & $5,3 \pm 2,9$ \\
\hline Admission en réanimation & $30(37)$ \\
\hline Mortalité à 28 jours & $28(35)$ \\
\hline \multicolumn{2}{|l|}{ Profils hémodynamiques par échocardiographie ${ }^{b}$} \\
\hline Hypovolémie sévère & $54(66)$ \\
\hline Insuffisance VG & $21(26)$ \\
\hline Hyperkinésie du VG avec vasoplégie & $26(32)$ \\
\hline Insuffisance VD & $5(10)$ \\
\hline Tamponnade & $0(0)$ \\
\hline Insuffisance valvulaire massive & $0(0)$ \\
\hline Aucune anomalie hémodynamique pertinente & $9(11)$ \\
\hline
\end{tabular}

Les résultats sont présentés en moyenne \pm écart-type. Les nombres entre parenthèses représentent les pourcentages. VCI : veine cave inférieure ; VG : ventricule gauche ; VD : ventricule droit ; SOFA : Sepsis-related Organ Failure Assessment. ${ }^{\text {a }}$ Volume médian de remplissage vasculaire au moment de l'échocardiographie. ${ }^{\mathrm{b}}$ Les mécanismes peuvent être associés 
vasoplégie avec hyperkinésie du VG chez 26 patients (32\%), dont six patients ( $8 \%$ ) sans signes d'hypovolémie associée, et l'insuffisance VG chez 21 patients (26\%) qui était associée à une insuffisance VD chez cinq patients. Aucun patient n'avait de dysfonction VD isolée ni de cœur pulmonaire aigu. Neuf patients avaient des anomalies mais non significatives et ont été considérés comme ayant un profil normal par absence d'identification d'un mécanisme de défaillance hémodynamique échographiquement pertinent. Parmi eux, cinq patients présentaient des signes cliniques de défaillance circulatoire (marbrures, encéphalopathie, baisse de la pression artérielle moyenne). Néanmoins, ils étaient moins tachycardes, avaient un score SOFA plus faible et une lactatémie inférieure aux autres patients (fréquence cardiaque : $94 \pm 15 \mathrm{bpm}$; pression artérielle moyenne : $64 \pm 10 \mathrm{mmHg}$; score SOFA : $3,5 \pm 1,7$; lactates : $2,6 \pm 1,2 \mathrm{mmol} / \mathrm{l})$. Aucun patient n'avait d'insuffisance valvulaire massive ni de tamponnade. Contrairement aux critères et mesures échocardiographiques, aucune différence significative n'a été trouvée entre les patients présentant les trois profils hémodynamiques concernant les critères cliniques, les paramètres de gravité (SOFA $p=0,55$; lactates $p=$ 0,68 ), l'orientation à la sortie du SU (réanimation $p=0,27$ ) et le pronostic (choc septique $p=0,16$ et mortalité à $\mathrm{j} 28 p=$ 0,63) (Tableau 3).

Les patients hypovolémiques et/ou hyperkinétiques étaient caractérisés respectivement par un diamètre maximal de VCI de $13 \pm 4$ et $13 \pm 6 \mathrm{~mm}$ associé à un collapsus inspiratoire dans 60 et $70 \%$ des cas, un rapport VD/VG inférieur à 0,8 et une cavité VG de petite taille (diamètre télédiastolique : $46 \pm 9$ et $48 \pm 7 \mathrm{~mm}$, respectivement). À l'inverse, les patients présentant une insuffisance VG précoce, isolée ou associée à une insuffisance $\mathrm{VD}$, avaient un diamètre téléexpiratoire de VCI moyen plus élevé $(18 \pm 6$ et
$19 \pm 2 \mathrm{~mm}$, respectivement), un diamètre télédiastolique du VG moyen également plus élevé ( $54 \pm 8$ et $53 \pm 7 \mathrm{~mm}$, respectivement), et un rapport VD/VG respectivement de 0,77 $\pm 0,16$ et $0,95 \pm 0,1$.

L'impact thérapeutique potentiel de l'évaluation échocardiographique concernait 21 patients $(26 \%)$ : poursuite $d u$ remplissage vasculaire au-delà de $30 \mathrm{ml} / \mathrm{kg}$ et adjonction d'un traitement vasopresseur précoce devant une vasoplégie importante pour 13 patients, et inversement arrêt prématuré du remplissage vasculaire pour huit patients (après $850 \pm$ $740 \mathrm{ml}$ ) avec introduction d'un inotrope positif pour trois patients (Fig. 1).

\section{Discussion}

L'étude présente confirme que la majorité des patients chez qui un sepsis est identifié au SU sont principalement hypovolémiques et vasoplégiques, mais montre qu'un quart d'entre eux présentent une insuffisance ventriculaire (gauche ou droite) précoce. L'impact thérapeutique potentiel de cette évaluation hémodynamique précoce par échocardiographie ciblée modifiait le standard de soins reposant sur les recommandations de la SSC chez 21 patients (26\%).

Le profil hémodynamique identifié chez un patient septique dépend de nombreux facteurs tels que le délai de l'évaluation, la fonction cardiaque sous-jacente, l'intensité de la réponse inflammatoire et le volume de remplissage vasculaire déjà reçu par le patient [16]. Dans l'étude présente, tous les patients ont été évalués très précocement au $\mathrm{SU}$, alors que le remplissage vasculaire médian déjà réalisé n'excédait pas $500 \mathrm{ml}$, c'est pourquoi le profil hypovolémique - associé ou non à un profil vasoplégique - était prépondérant (66 \%). C'est la raison pour laquelle la VCI de petite taille

Tableau 3 Comparaison des critères de gravité entre les sous-groupes de patients septiques répartis selon le mécanisme de défaillance hémodynamique initiale identifiée en échocardiographie

\begin{tabular}{|c|c|c|c|c|}
\hline & $\begin{array}{l}\text { Hypovolémie sévère } \\
(n=54)\end{array}$ & $\begin{array}{l}\text { Vasoplégie } \\
\text { avec hyperkinésie du VG } \\
(n=26)\end{array}$ & $\begin{array}{l}\text { Insuffisance VG } \\
(n=21)\end{array}$ & Valeur de $p$ \\
\hline Âge (années) & $71 \pm 13$ & $67 \pm 14$ & $73 \pm 15$ & 0,36 \\
\hline Homme & $30(55)$ & $13(50)$ & $16(76)$ & 0,16 \\
\hline Choc septique & $26(48)^{\mathrm{a}}$ & $16(62)^{\mathrm{a}}$ & $7(33)$ & 0,16 \\
\hline Lactate $(\mathrm{mmol} / \mathrm{l})$ & $4,7 \pm 4,6$ & $5,4 \pm 5,8$ & $4,2 \pm 2,5$ & 0,68 \\
\hline Score SOFA moyen & $5,5 \pm 3,0$ & $6,1 \pm 3,6$ & $5,1 \pm 3,1$ & 0,55 \\
\hline Admission en réanimation & $17(31)$ & $13(50)$ & $8(38)$ & 0,27 \\
\hline Mortalité à 28 jours & $19(35)$ & $8(31)$ & $5(24)$ & 0,63 \\
\hline
\end{tabular}



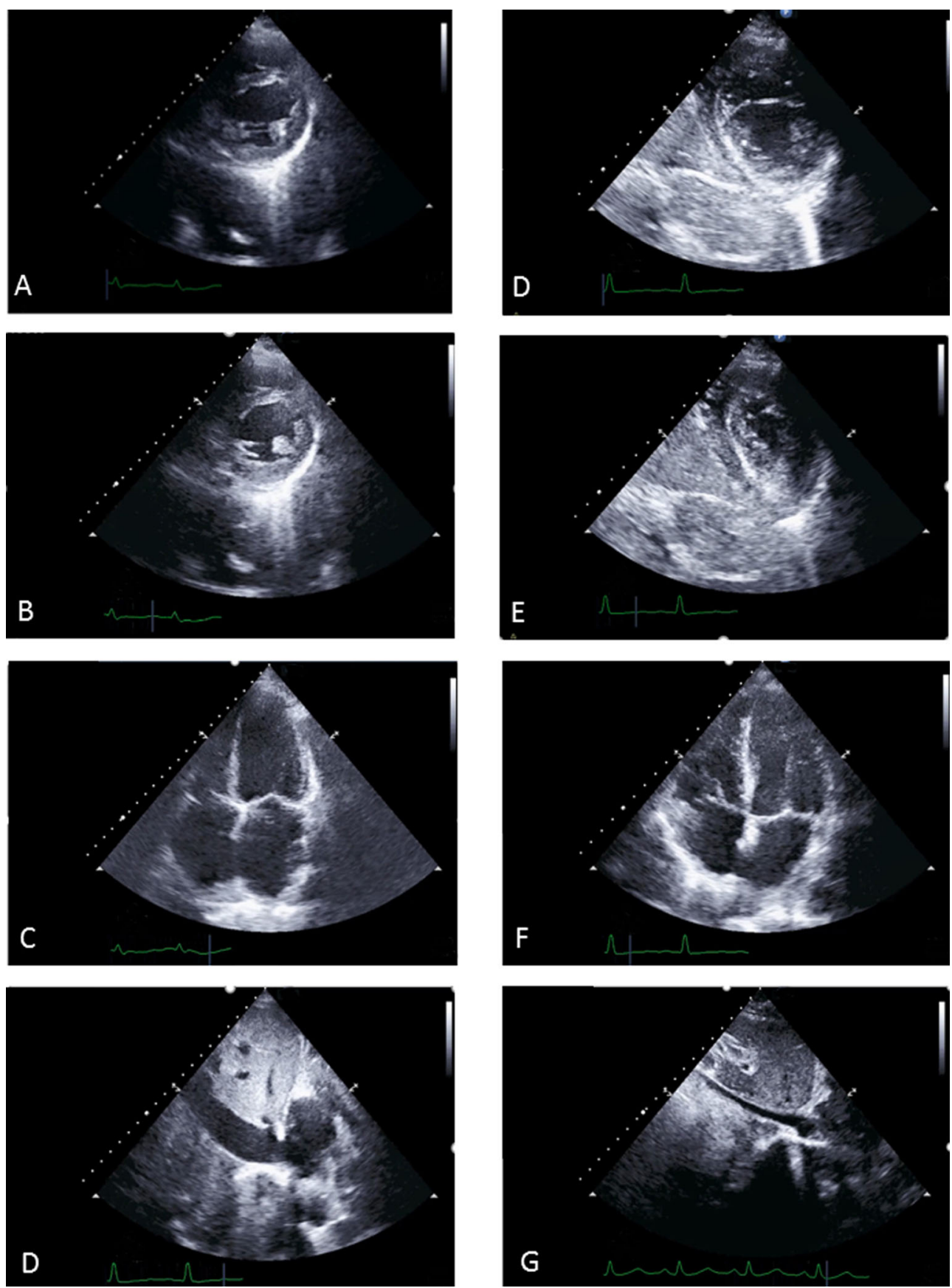

Fig. 1 Coupes échocardiographiques illustrant les différents profils selon le mécanisme de défaillance hémodynamique initial (les deux patients étaient infectés avec un score qSOFA $\geq 2$ points dont l'hypotension artérielle) Cas 1 (panneaux gauches) : patient de 71 ans, pneumopathie franche lobaire aiguë droite, avec une dysfonction systolique du ventricule gauche majeure en vue parasternale petit axe (peu de modifications visuelles de la taille de la cavité ventriculaire gauche entre la télédiastole (A) et la télésystole [B]), sans anomalie structurale en faveur d'une cardiopathie sous-jacente visible en vue apicale quatre cavités (C), associée à une dilatation de la veine cave inférieure mesurée à $28 \mathrm{~mm}$ en vue sous-costale (D) en faveur d'une congestion veineuse systémique Cas 2 (panneaux droits) : patiente de 46 ans se présentant avec un urosepsis et une hypovolémie sévère associée à une vasoplégie comme reflétée par une hyperkinésie du ventricule gauche en vue parasternale petit axe (fraction de réduction de surface du VG estimée visuellement $>60 \%$ ) avec disparition presque complète de la cavité ventriculaire gauche entre la télédiastole (D) et la télésystole (E). Là aussi, aucune cardiopathie évidente n'est visuellement identifiée en vue apicale quatre cavités (F). À noter la diminution importante de la taille de la veine cave inférieure à $8 \mathrm{~mm}(\mathrm{G})$ avec collapsus inspiratoire complet en temps réel 
avec un collapsus inspiratoire marqué et l'hyperkinésie du VG étaient les signes échocardiographiques les plus observés, comme précédemment rapporté chez des patients se présentant au SU avec une hypotension artérielle d'étiologie inconnue [17]. Le remplissage vasculaire est un pilier de la prise en charge initiale des patients septiques afin de corriger l'hypovolémie absolue et relative et d'optimiser la perfusion tissulaire [18]. Le respect des bundles de la SSC améliore la survie des patients sans que soit clairement détaillé l'impact de chaque composante thérapeutique qui la compose $[2,19]$. Notamment, plusieurs études remettent en cause le bénéfice $\mathrm{du}$ remplissage vasculaire uniforme et systématique de $30 \mathrm{ml} / \mathrm{kg}[3,20]$, dont le niveau de preuve est très faible et actuellement débattu [21]. En effet, une telle approche ne prend pas en compte le profil hémodynamique précoce du patient septique, et notamment pas la présence d'une cardiopathie sous-jacente connue. Dans l'étude présente, $66 \%$ des patients avaient un profil hypovolémique associé ou non à un profil vasoplégique, et donc étaient éligibles au remplissage vasculaire préconisé par la SSC. Néanmoins, un remplissage vasculaire supérieur à $30 \mathrm{ml} / \mathrm{kg}$ peut être nécessaire chez les patients présentant une hyperkinésie importante du VG qui reflète une vasoplégie sous-jacente, comme réalisé devant les données échocardiographiques chez 13 de nos patients, en adjonction à l'introduction de vasopresseurs. La forte prévalence de ce profil hémodynamique dans notre cohorte est probablement liée à l'absence de traitement vasopresseur visant à corriger la vasoplégie sous-jacente au moment de l'échocardiographie précocement réalisée au SU [22].

Parmi les mécanismes physiopathologiques multiples, complexes et évolutifs dans le temps à l'origine de la défaillance circulatoire du sepsis [6], une atteinte intrinsèque de la contractilité myocardique est fréquemment observée et communément appelée « cardiomyopathie septique » [15]. En l'absence de définition consensuelle actuellement, sa prévalence est estimée entre 40 et $60 \%$ des patients en choc septique [14]. La cardiomyopathie septique se distingue de l'insuffisance cardiaque congestive classique sur le fait qu'elle ne s'accompagne généralement pas d'une élévation des pressions de remplissage gauche [23]. De plus, ces patients n'augmentent habituellement pas leur débit cardiaque suite au remplissage vasculaire et sont à haut risque de positiver indûment leur bilan hydrique, ce qui est potentiellement néfaste sur le pronostic. Chez les patients en choc septique évalués par échocardiographie en réanimation après optimisation du remplissage vasculaire et mise sous vasopresseur, la dysfonction systolique du VG induite par le sepsis peut être présente dès le premier jour ou apparaître deux, voire trois jours après l'admission. Dans l'étude présente, $26 \%$ des patients présentaient une insuffisance VG attribuable à une cardiomyopathie septique précoce, cinq d'entre eux ayant une insuffisance VD associée. Ce résultat confirme ceux rapportés dans notre expérience initiale, avec une prévalence de la dysfonction VG et/ou VD de $31 \%$ chez les patients septiques évalués précocement par échocardiographie niveau ECMU 1 dès leur admission au SU [24]. Chez huit de ces patients, le remplissage vasculaire a été interrompu après une moyenne de $850 \pm 740 \mathrm{ml}$ (soit environ $10 \mathrm{ml} / \mathrm{kg}$ ), sans respecter le volume de $30 \mathrm{ml} / \mathrm{kg}$ préconisé [19]. Trois de ces patients ont en outre bénéficié précocement d'un traitement par inotrope positif sur les données échocardiographiques [25]. Actuellement, le gain pronostique de l'utilisation de dobutamine chez les patients en choc septique n'a pas été démontré par une étude randomisée contrôlée. La dobutamine est donc proposée lorsqu'une hypoxie tissulaire persistante est attribuée à une insuffisance cardiaque sévère chez un patient en choc septique sous vasopresseur et dont le remplissage vasculaire a été préalablement optimisé [19]. Finalement, aucun cas de tamponnade ou d'insuffisance valvulaire aiguë sévère n'a été observé dans notre cohorte, car les épanchements péricardiques purulents compressifs et les endocardites infectieuses mutilantes restent peu fréquemment à l'origine d'un choc septique [26].

L'échocardiographie est recommandée en première intention dans l'évaluation des patients qui présentent une insuffisance circulatoire aiguë et nécessitent une évaluation hémodynamique [7]. L'impact thérapeutique d'une évaluation hémodynamique précoce par échocardiographie ciblée (niveau ECMU 1) dès l'admission des patients septiques au SU est encore peu décrit. Dans une cohorte de petite taille, Sekiguchi et al. [27] ont rapporté un impact thérapeutique pouvant atteindre $27 \%$ des patients septiques au cours des six heures de prise en charge initiale. Dans une étude réalisée sur 118 patients en état de choc d'étiologie diverse au SU, Shokoohi et al. [28] décrivent l'intérêt de l'échocardiographie précoce à la fois en termes diagnostique et thérapeutique, montrant un impact dans $15 \%$ des cas concernant l'instauration d'un traitement vasopresseur et l'adaptation du remplissage vasculaire. L'utilisation de l'échocardiographie semble donc prometteuse pour optimiser la prise en charge des patients septiques dès leur admission au SU. Une analyse sur base de données a récemment rapporté une diminution de la mortalité à 28 jours chez les patients septiques dont le traitement a été guidé par échocardiographie [29]. De plus, Kanji et al. [30] ont montré dans une étude rétrospective non randomisée de type avant/après que l'évaluation par échocardiographie ciblée (niveau basique) des patients présentant une hypotension artérielle était associée à une amélioration de la survie à 28 jours. Néanmoins, l'impact d'une telle stratégie diagnostique précoce sur le pronostic des patients septiques reste à déterminer par des études prospectives multicentriques de plus grande échelle.

Notre étude présente plusieurs limites. Premièrement, le recrutement des patients n'a pas été exhaustif, car il dépendait de la disponibilité des investigateurs pour réaliser 
l'échocardiographie précoce. Néanmoins, ce biais de sélection a été aléatoire et le nombre de sujets nécessaires déterminé a priori a été atteint. Deuxièmement, nous avons identifié les profils hémodynamiques uniquement par évaluation bidimensionnelle visuelle (niveau ECMU 1), sans mesure doppler, ce qui n'a pas permis de mesurer ou d'évaluer indirectement certains paramètres hémodynamiques tels que et le débit cardiaque et les pressions de remplissage du VG. Ces critères auraient pu permettre de mieux différencier les profils notamment en cas d'hypovolémie et de vasoplégie, dont le chevauchement est fréquent. Cependant, le niveau ECMU 1 permet d'identifier différents profils hémodynamiques permettant de guider une approche thérapeutique personnalisée, en accord avec les préconisations de la SSC concernant les axes prioritaires de recherche [5]. Troisièmement, l'évaluation par échocardiographie était ponctuelle et non répétée, ce qui n'a pas permis d'évaluer l'efficacité et la tolérance des interventions thérapeutiques réalisées suite à l'évaluation hémodynamique précoce. Quatrièmement, l'impact pronostique de l'évaluation hémodynamique par échocardiographie ciblée n'a pas été étudié dans cette étude observationnelle, mais devra l'être par un essai randomisé multicentrique comparant une stratégie thérapeutique guidée par échocardiographie et le standard de soins reposant sur les recommandations de la SSC.

\section{Conclusion}

L'évaluation des patients septiques par échocardiographie ciblée au SU (ECMU 1) identifie principalement un profil hypovolémique et vasoplégique. L'identification d'une dysfonction cardiaque précoce chez environ un quart des patients semble remettre en question la stratégie thérapeutique initiale reposant sur un remplissage vasculaire de $30 \mathrm{ml} / \mathrm{kg}$ chez tous les patients. L'impact pronostique potentiel de la prise en charge thérapeutique précoce des patients septiques guidée par l'évaluation échocardiographique au SU reste à établir.

Liens d'intérêts : les auteurs déclarent ne pas avoir de liens d'intérêts.

\section{Références}

1. Reinhart K, Daniels R, Kissoon N, et al (2017) Recognizing sepsis as a global health priority - A WHO resolution. N Engl J Med 377:414-7

2. Levy MM, Evans LE, Rhodes A (2018) The Surviving Sepsis Campaign bundle: 2018 update. Crit Care Med 46:997-1000

3. Marik PE, Linde-Zwirble WT, Bittner EA, et al (2017) Fluid administration in severe sepsis and septic shock, patterns and out- comes: an analysis of a large national database. Intensive Care Med 43:625-32

4. Malbrain MLNG, Van Regenmortel N, Saugel B, et al (2018) Principles of fluid management and stewardship in septic shock: it is time to consider the four D's and the four phases of fluid therapy. Ann Intensive Care 8:66

5. Coopersmith CM, De Backer D, Deutschman CS, et al (2018) Surviving Sepsis Campaign: research priorities for sepsis and septic shock. Crit Care Med 46:1334-56

6. Aneman A, Vieillard-Baron A (2016) Cardiac dysfunction in sepsis. Intensive Care Med 42:2073-6

7. Cecconi M, De Backer D, Antonelli M, et al (2014) Consensus on circulatory shock and hemodynamic monitoring. Task force of the European Society of Intensive Care Medicine. Intensive Care Med 40:1795-815

8. Bouferrache K, Amiel JB, Chimot L, et al (2012) Initial resuscitation guided by the Surviving Sepsis Campaign recommendations and early echocardiographic assessment of hemodynamics in intensive care unit septic patients: a pilot study. Crit Care Med 40:2821-7

9. Shankar-Hari M, Phillips GS, Levy ml, et al (2016) Developing a new definition and assessing new clinical criteria for septic shock: for the third international consensus definitions for sepsis and septic shock (Sepsis-3). JAMA 315:775-87

10. Duchenne J, Martinez M, Rothmann C, et al (2016) Premier niveau de compétence pour l'échographie clinique en médecine d'urgence. Recommandations de la Société française de médecine d'urgence par consensus formalisé. Ann Fr Med Urgence 6:284-95

11. Labovitz AJ, Noble VE, Bierig M, et al (2010) Focused cardiac ultrasound in the emergent setting: a consensus statement of the American Society of Echocardiography and American College of Emergency Physicians. J Am Soc Echocardiogr 23:1225-30

12. Nagdev AD, Merchant RC, Tirado-Gonzalez A, et al (2010) Emergency department bedside ultrasonographic measurement of the caval index for non-invasive determination of low central venous pressure. Ann Emerg Med 55:290-5

13. Geri G, Vignon P, Aubry A, et al (2019) Cardiovascular clusters in septic shock combining clinical and echocardiographic parameters: a post hoc analysis. Intensive Care Med 45:657-67

14. Vieillard-Baron A, Cecconi M (2014) Understanding cardiac failure in sepsis. Intensive Care Med 40:1560-3

15. Beesley SJ, Weber G, Sarge T, et al (2018) Septic cardiomyopathy. Crit Care Med 46:625-34

16. Ricard-Hibon A, Losser MR, Kong R, et al (1998) Systemic pressure-flow reactivity to norepinephrine in rabbits: impact of endotoxin and fluid loading. Intensive Care Med 24:959-66

17. Atkinson PR, Milne J, Diegelmann L, et al (2018) Does pointof-care ultrasonography improve clinical outcomes in emergency department patients with undifferentiated hypotension? An international randomized controlled trial from the SHoC-ED investigators. Ann Emerg Med 72:478-89

18. Rivers E, Nguyen B, Havstad S, et al (2001) Early goal-directed therapy in the treatment of severe sepsis and septic shock. N Engl J Med 345:1368-77

19. Rhodes A, Evans LE, Alhazzani W, et al (2017) Surviving Sepsis Campaign: International guidelines for management of sepsis and septic shock: 2016. Crit Care Med 45:486-552

20. Seymour CW, Gesten F, Prescott HC, et al (2017) Time to treatment and mortality during mandated emergency care for sepsis. N Engl J Med 376:2235-44

21. Meyhoff TS, Møller MH, Hjortrup PB, et al (2020) Lower vs. higher fluid volumes during initial management of sepsis: a systematic review with meta-analysis and trial sequential analysis. Chest 157:1478-96 
22. Scheeren TWL, Bakker J, De Backer D, et al (2019) Current use of vasopressors in septic shock. Ann Intensive Care 9:20

23. Bloch A, Berger D, Takala J (2016) Understanding circulatory failure in sepsis. Intensive Care Med 42:2077-9

24. Lafon T, Appert A, Hadj M, et al (2020) Comparative early haemodynamic profiles in patients presenting to the emergency department with septic and non-septic acute circulatory failure using focused echocardiography. Shock 53:695-700

25. Kakihana Y, Ito T, Nakahara M, et al (2016) Sepsis-induced myocardial dysfunction: pathophysiology and management. J Intensive Care 4:22

26. Chou EH, Mann S, Hsu TC, et al (2020) Incidence, trends, and outcomes of infection sites among hospitalizations of sepsis: a nationwide study. PloS One 15:e227752
27. Sekiguchi H, Harada Y, Villarraga HR, et al (2017) Focused cardiac ultrasound in the early resuscitation of severe sepsis and septic shock: a prospective pilot study. J Anesth 31:487-93

28. Shokoohi H, Boniface KS, Pourmand A, et al (2015) Bedside ultrasound reduces diagnostic uncertainty and guides resuscitation in patients with undifferentiated hypotension. Crit Care Med 43:2562-9

29. Feng M, McSparron JI, Kien DT, et al (2018) Transthoracic echocardiography and mortality in sepsis: analysis of the MIMIC-III database. Intensive Care Med 44:884-92

30. Kanji HD, McCallum J, Sirounis D, et al (2014) Limited echocardiography-guided therapy in subacute shock is associated with change in management and improved outcomes. J Crit Care 29:700-5 